\title{
Composición y sobreposición dietaria de Pinguipes chilensis (Perciformes: Pinguipedidae), Cheilodactylus variegatus (Perciformes: Cheilodactylidae) y Aplodactylus punctatus (Perciformes: Aplodactylidae) en el litoral costero valdiviano, Chile
}

\author{
Dietary composition and overlap of Pinguipes chilensis (Perciformes: Pinguipedidae), \\ Cheilodactylus variegatus (Perciformes: Cheilodactylidae) and Aplodactylus punctatus \\ (Perciformes: Aplodactylidae) in the Valdivian coast, Chile
}

\author{
María Fernanda Cornejo-Acevedo ${ }^{1}$, Pablo Fierro $^{1,2,3}$, Carlos Bertrán $^{1}$ \& Luis Vargas-Chacoff ${ }^{*}$ \\ ${ }^{1}$ Instituto de Ciencias Marinas y Limnológicas, Facultad de Ciencias, Universidad Austral de Chile, Independencia 641, \\ Valdivia, Chile. \\ ${ }^{2}$ Departamento de Zoología, Facultad de Ciencias Naturales y Oceanográficas, Universidad de Concepción, Víctor Lamas \\ 1290, Concepción, Chile.
}

${ }^{3}$ Centro de Ciencias Ambientales EULA-CHILE, Universidad de Concepción, Casilla 160-C, Concepción, Chile.

luis.vargas@uach.cl

\begin{abstract}
RESUMEN
Pinguipes chilensis, Cheilodactylus variegatus y Aplodactylus punctatus son especies comunes de ser registradas a lo largo de la costa chilena, no obstante información sobre su alimentación es escasa para la zona sur de nuestro país. Por ello, se analizó la composición y sobreposición dietaria de estas especies para la costa Valdiviana. Los resultados señalan que para el periodo primaveral del año 2010, en $P$. chilensis $(n=41)$ se identificaron 20 presas, todas caracterizadas por ser invertebrados de gran tamaño, principalmente crustáceos $(55 \%)$ y moluscos $(40 \%)$, siendo la especie Homalaspis plana la más representativa (IRI 70\%). En Ch. variegatus $(\mathrm{n}=38)$ se identificaron 37 presas, mayoritariamente pequeños invertebrados, principalmente crustáceos $(49 \%)$, moluscos $(31 \%)$ y anélidos $(10 \%)$. Dentro de estos últimos dominaron los poliquetos de la Familia Nereidae (IRI 23,2\%). En A. punctatus $(\mathrm{n}=32)$ la diversidad de algas rojas $(50 \%)$ predominó sobre las pardas (41\%) y verdes (9\%), siendo identificadas 34 especies presa, donde Colpomenia sp. (Phaeophyta) fue la más representativa (IRI 32,4\%). El análisis de sobreposición de dieta entre las tres especies mostró una baja similitud entre las dietas. Las tres especies analizadas en este estudio, se caracterizan por compartir un mismo hábitat. P. chilensis y Ch. variegatus se muestran ambas como depredadores carnívoros generalistas, ya que las dos especies presentan un amplio espectro alimentario sin embargo la morfología y el tamaño de su boca influirían en el tamaño de la presa que consumen. En cambio, A. punctatus se confirma como una especie herbívora especialista, ya que consume preferentemente algas rojas.
\end{abstract}

Palabras clave: Alimentación, ítemes- presas, litoral costero Valdiviano.

\begin{abstract}
Pinguipes chilensis, Cheilodactylus variegatus and Aplodactylus punctatus are common species in Chilean coastal, but currently information on dietary composition in south of Chile is scarce. Therefore was analyzed the dietary composition and diet overlap of theses species in the coast of Valdivia. The results indicated in spring 2010 that for P. chilensis ( $\mathrm{n}=41)$ 20 preys were identified, all characterized as being oversized invertebrates, mainly crustaceans (55\%) and molluscs (40\%), being the Homalaspis plana the most representative specie (70\% IRI value). In Ch. variegatus $(\mathrm{n}=38) 37$ preys were identified, mostly small invertebrates, mainly crustaceans (49\%), molluscs (31\%) and annelids (10\%). In the polychaetes of Nereidae Family was most common (23.2\% IRI value). In A. punctatus ( $\mathrm{n}=32)$ red algae predominated $(50 \%)$ over the brown (41\%) and green algae (9\%), in total 34 prey species were identified, where Colpomenia sp. (Phaeophyta) was the most common prey (32.4\% IRI). Analysis of diet overlap among the three species showed a low similarity between diets. The three species in this study are characterized by sharing the same habitat. P. chilensis and Ch. variegatus shown both as generalist carnivores, since both species have a wide food spectrum, however the morphology and size of your mouth influence the size of the prey they consume. Instead, A. punctatus was confirmed as a specialist herbivore species, consuming preferably red algae.
\end{abstract}

KEYwords: Feeding, items-preys, coastal of Valdivia. 


\section{INTRODUCCION}

El comportamiento alimentario es característico de cada especie y muchos autores han puesto énfasis en estudiar los factores de los cuales depende el fenómeno de la selectividad sobre un particular tipo de alimento por parte de los peces (Moreno \& Zamorano 1980; Bertrán et al. 2013).

En la literatura se han planteado diferentes agentes como determinantes en los procesos de selección de dieta (Cáceres et al. 1993), entre los factores externos al pez, está la relación entre el tamaño de la presa y los cambios ontogénicos del depredador (Fierro et al. 2014), el grado de movilidad de la presa y su visibilidad, la disponibilidad de la presa en el medio y ciertos factores internos del depredador como el grado de hambre, ritmos circadianos, campo de visión, experiencia del pez y ritmos anuales afectados por procesos fisiológicos. Muchos de los factores que tienen relación con la selectividad, también tienen relación con el valor energético de la presa, la eficiencia de asimilación, el contenido de nutrientes y principalmente la disponibilidad relativa del alimento; que son factores que también pueden influir en la selección de este y que han sido incorporados en modelos teóricos de balance energético costo-beneficio en la teoría de las estrategias de alimentación (Botero 2004). La selección de un alimento en particular, repercute sobre la estructura de la comunidad, incluso cuando la presión de depredación no es muy alta. De ahí, el alto interés ecológico que presenta el estudio de la selectividad de la presa por parte de un depredador (Moreno \& Zamorano 1980).

En Chile, existe la necesidad de incrementar el conocimiento básico sobre la biología de los peces (García \& Chong 2002), especialmente aquellos que tienen importancia económica para nuestro país, debido a que están sometidas a una intensa presión de pesca. Investigar sobre su conducta alimentaria, y así obtener modelos de estudio, conocer aspectos de su explotación y conservación (Jiménez 2006) se vuelven entonces un objetivo de vital importancia.

El "rollizo" Pinguipes chilensis (Valenciennes 1833) se distribuye en Chile desde las costas de Arica hasta el extremo austral de Chile, siendo una especie endémica del Océano Pacifico Suroriental (Navarro \& Pequeño 1979; Pequeño et al. 1995). Posee importancia económica a escala artesanal siendo capturada preferentemente en el litoral norte y centro de Chile (SERNAPESCA 2011). El hábitat de $P$. chilensis es el intermareal y submareal, con fondos rocosos para los adultos y arenosos para los juveniles (Moreno \& Zamorano 1980; Pequeño \& Lamilla 1995). Su distribución batimétrica fluctúa entre los 0 y $40 \mathrm{~m}$ de profundidad, aunque su principal rango de distribución varía entre los $5 \mathrm{y}$ 15 m (González 2001). La dieta de esta especie esta basada en organismos bentónicos como crustáceos, moluscos o poliquetos (Vargas et al. 1999; González \& Oyarzún 2003).

El "bilagay" o "pintacha" Cheilodactylus variegatus (Valenciennes 1833) se distribuye desde Bahía Metri (Región de los Lagos) por el sur hasta Arica por el norte. La información científica relativa a esta especie es escasa (Vargas \& Pequeño 2001). Su distribución batimétrica, alcanza hasta los $20 \mathrm{~m}$ de profundidad, y está asociada a la franja litoral entre rocas cubiertas de algas. La dieta de esta especie, al igual que la de $P$. chilensis esta compuesta principalmente de crustáceos y moluscos bentónicos (Moreno \& Flores 2002).

La "jerguilla" Aplodactylus punctatus (Valenciennes 1832) es considerada según Cáceres et al. (1993) una de las especies más abundantes del ensamble de peces litorales de Chile central. Su distribución en Chile abarca desde Arica por el norte hasta el Golfo de Arauco por el sur (Chirichigno 1998). Habita la zona intermareal rocosa ocupando preferentemente hábitats asociados a manchones de algas pardas y verdes. Su distribución batimétrica alcanza el límite impuesto por la presencia del último cordón de algas pardas (aproximadamente $20 \mathrm{~m}$ de profundidad). $\mathrm{La}$ dieta de esta especie es básicamente herbívora, compuesta principalmente por algas rojas, pardas y verdes, las cuales variarían en representatividad de acuerdo a la zona de estudio (Cáceres et al. 1993).

Cada una de las especies mencionadas, comúnes en las costas chilenas, están principalmente descritas para el norte y centro del país. La información sobre su alimentación es escasa para la zona sur de Chile, por lo que el objetivo principal de este trabajo fue analizar el contenido estomacal de ejemplares de $P$. chilensis, Ch. variegatus y A. punctatus; conocer el grado de sobreposición de sus dietas y discutir los posibles factores que incidirían en sus preferencias alimentarias en la costa del sur de Chile.

\section{MATERIALES Y METODOS}

El presente trabajo se llevó a cabo en el litoral de la provincia de Valdivia, región de Los Ríos, Chile; en el sector de Punta Morro Gonzalo ( $39^{\circ} 51^{`} 13,51^{\prime \prime}$ S 73⒉ $\left.8^{\prime} 18,14 ” W\right)$ (Fig.1), ubicado al norte de la localidad de Chaihuín, cercano a la bahía de Corral. La zona submareal se caracteriza por la presencia de fondos rocosos hasta aproximadamente los $40 \mathrm{~m}$ de profundidad, seguido de un substrato arenoso y la presencia de extensos bosques del alga parda Macrocystis pirifera entre los 2 y los $20 \mathrm{~m}$ de profundidad.

Durante los días 5 y 6 de noviembre del año 2010, se capturaron ejemplares de Pinguipes chilensis, Cheilodactylus variegatus y Aplodactylus punctatus 


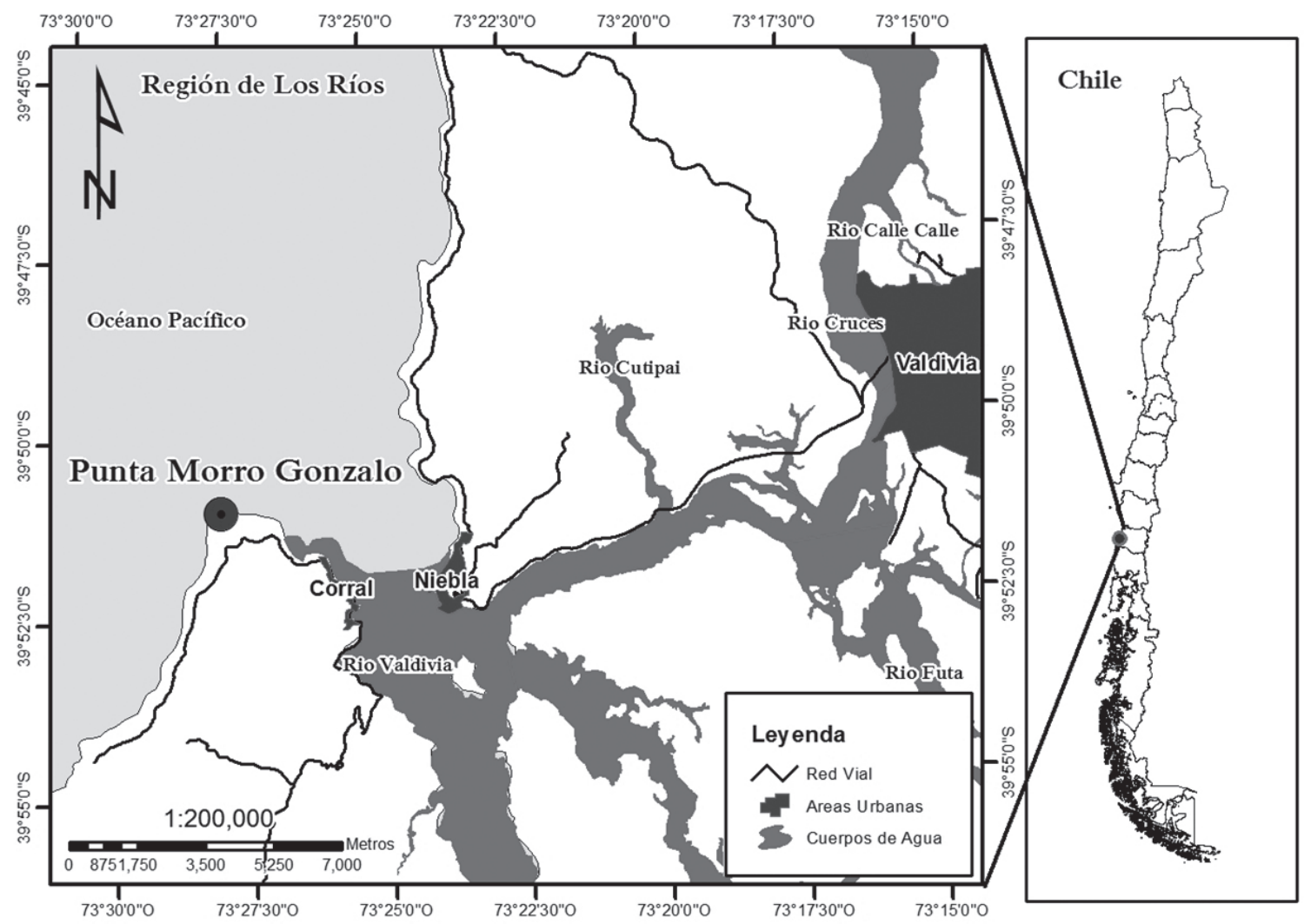

Figura 1. Mapa del área de estudio ( 3951 '13,51”S, 73²8’18,14”W). Punto negro, zona de muestreo.

FigURE 1. Map of the study area $\left(\sim 39^{\circ} 51^{\prime} 13.51^{\prime \prime}\right.$ S, $\left.73^{\circ} 28^{\prime} 18,14 ” \mathrm{~W}\right)$. Black dot is the sampling area.

mediante arpón por buceo apnea entre 1 y $20 \mathrm{~m}$ de profundidad, aproximadamente a $20-30 \mathrm{~m}$ de la orilla de la playa. Los ejemplares capturados fueron medidos con una cinta métrica de $0,1 \mathrm{~cm}$ de precisión para obtener la longitud total (LT), y pesados mediante una balanza de terreno graduada. Los peces fueron eviscerados y el estómago completo fue fijado en alcohol al $90 \%$ para ser trasladados al Instituto de Ciencias Marinas y Limnológicas de la Universidad Austral de Chile, donde se les extrajo el contenido estomacal, el que fue preservado en alcohol al $70 \%$ para su posterior análisis.

Los contenidos estomacales fueron analizados bajo lupa binocular estereoscópica y diferenciando tratamientos acorde a la especie. Particularmente para la especie $C h$. variegatus, se utilizó la metodología de homogenización por "cuarteo", debido a la alta abundancia de contenido estomacal y principalmente aplicada para muestras de zooplancton (UNESCO 1968), así los diferentes ítemes alimentarios encontrados fueron identificados, separados y contados, multiplicando la cantidad presente por el número de la cantidad de cuarteos que se realizaron en la muestra, obteniendo así los datos de abundancia total por estómago. Para $P$. chilensis, los diferentes ítemes alimentarios fueron identificados, contados y separados, obteniendo datos de abundancia total por cada estomago analizado Hyslop (1980). Para A. punctatus los diferentes ítemes alimentarios fueron identificados y separados hasta el nivel taxonómico más bajo posible, debido a la alta prevalencia de algas, los ítemes alimentarios fueron secados en estufa a $60^{\circ} \mathrm{C}$ por $12 \mathrm{~h}$, obteniendo así los datos de biomasa, de acuerdo a la metodología de Hyslop (1980) y modificada por Griffiths (1997).

El análisis y la determinación de los ítemes presa se realizó con el empleo de claves de identificación, para crustáceos y moluscos (Zagal \& Hermosilla 2001), poliquetos (Fauchald 1977), anfípodos (Barnard 1969), isópodos (Menzies 1962) y algas (Santelices 1989). Las diferencias entre las longitudes totales y entre los pesos totales de los individuos de las tres especies analizadas se evaluaron utilizando el test no paramétrico de Kruskall Wallis (Siegel \& Castellan 1988), empleando el programa STATGRAPHICS PLUS 5.1. 
Para evaluar la contribución particular de cada presa, se calculó el índice de importancia relativa, establecido por Pinkas et al. (1971) y usado por numerosos autores (e.g. González \& Oyarzún 2003; Bertrán et al. 2013; Fierro et al. 2014), expresando el valor de importancia relativa de los ítems contenidos en cada estómago del predador, de la siguiente manera:

$$
I R I=\frac{(\% \mathrm{~F} * \% N)}{100}
$$

Tanto para aquellos casos cuantificables; y para el caso de los ítems no cuantificables, el IRI fue calculado de acuerdo a lo postulado por Griffiths (1997) donde:

$$
I R I=\frac{(\% \mathrm{~F} \cdot \% V)}{100}
$$

Donde IRI es el índice de importancia relativa, $\% F$ frecuencia de ocurrencia de taxa en el estómago analizado, $\% \mathrm{~N}$ la abundancia relativa $\mathrm{y} \% \mathrm{~V}$ el volumen relativo. Ambos expresados en porcentaje para facilitar la comparación entre los diferentes ítemes encontrados.

Para calcular el índice de sobreposición de dieta entre las tres especies, se calculó el Índice de sobreposición de Pianka (1973), calculado de la siguiente manera:

$$
\mathrm{O} v=\frac{\Sigma \text { piqi }}{\sqrt{\left(\Sigma \mathrm{pi}^{2} \Sigma \mathrm{qi}^{2}\right)}}
$$

Donde Ov es el índice de sobreposición entre las tallas p y q, donde pi y qi son las frecuencias relativas con que un par cualquiera de especies usa los recursos i. Este índice varía entre 0 y 1 (siendo mayor cuando la dieta es similar). Se definió una sobreposición significativa de dietas (considerando valores $>0,6$ ) siguiendo a Fierro et al. (2014).

\section{RESULTADOS}

\section{Parámetros Biológicos}

Se capturaron 41 individuos de $P$. chilensis, , el promedio de la longitud total (LT) fue de 39,6 $\pm 4,0 \mathrm{~cm}$, con un valor mínimo de $29 \mathrm{~cm}$ y un valor máximo de $47 \mathrm{~cm}$. Para el caso de Ch. variegatus un total de 38 individuos fueron capturados, siendo el promedio de la longitud total de 30,1 $\pm 1,5 \mathrm{~cm}$ con valores entre 27 y $33,5 \mathrm{~cm}$ de longitud total. Finalmente para la especie A. punctatus se capturaron 32 individuos, siendo el promedio de LT de $34,4 \pm 2,5 \mathrm{~cm}$ con valores entre los 25 y $38 \mathrm{~cm}$ (Tabla 1, Fig. 2a). Se registraron diferencias estadísticamente significativas entre las longitudes totales (test Kruskall-Wallis; $\mathrm{H}=75,96$, $P<0,05)$ de las tres especies analizadas con un nivel de confianza del $95 \%$.
(A)

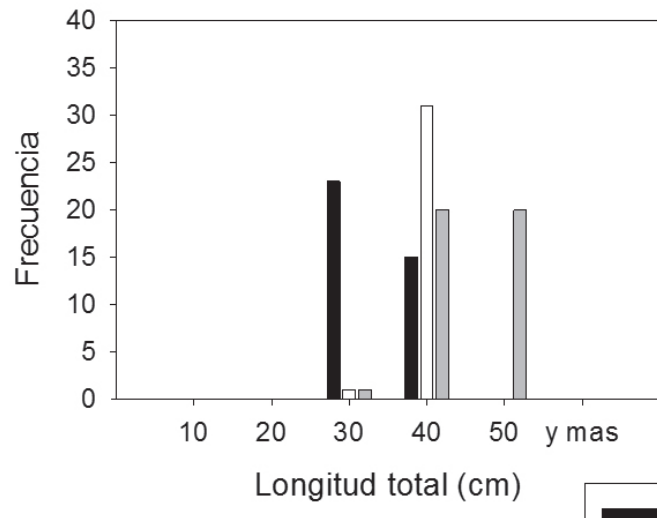

(B)

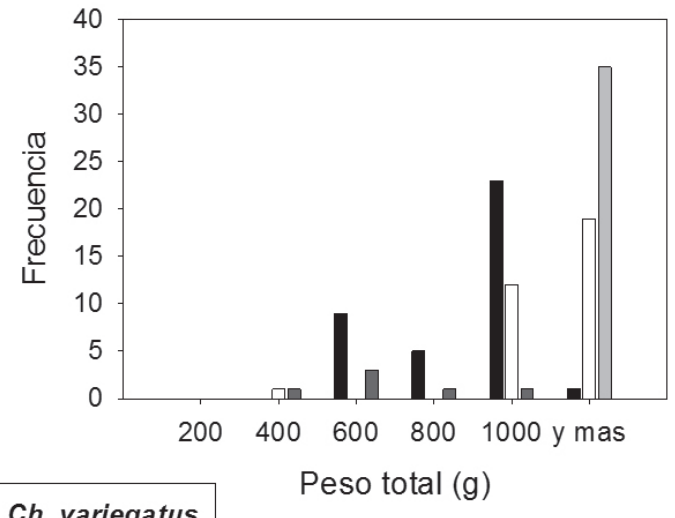

variegatus

A. punctatus

P. chilensis

Figura 2. A) Distribución de la frecuencia de tallas (cm) y B) distribución de la frecuencia de pesos ( $\mathrm{g}$ ) de Pinguipes chilensis, Cheilodactylus variegatus y Aplodactylus punctatus, en noviembre del 2010.

Figure 2. A) Distribution of length frequencies (cm) and B) the frequency distribution of weights ( $\mathrm{g}$ ) of Pinguipes chilensis, Cheilodactylus variegates and Aplodactylus punctatus, in November 2010. 
El peso total de los individuos analizados promedió 1453,7 $\pm 441,6$ g para $P$. chilensis, con un valor mínimo de $400 \mathrm{~g}$ y un máximo de $2500 \mathrm{~g}$; mientras que para Ch. variegatus el promedio del peso total fue de $852,6 \pm 179,7 \mathrm{~g}$, con valores mínimos de 500 g y máximos de 1300 g. Para A. punctatus, $e$ promedio del peso total fue de $1193,8 \pm 271,1$ g (Fig. 2b), con valores mínimos y máximos que van desde los 400 hasta los $1600 \mathrm{~g}$, respectivamente (Tabla 1). Al igual que para la longitud total, se registraron diferencias estadísticamente significativas entre los pesos totales (test Kruskall-Wallis; $\mathrm{H}$ $=48,23, p$-valor $<0,05)$ de las tres especies analizadas, con un nivel de confianza del $95 \%$.
ANÁLISIS CONTENIDO ESTOMACAL

En las tres especies analizadas se observó un alto porcentaje de estómagos con contenido (Tabla 1). Para P. chilensis se analizó un total de 40 estómagos en los que se identificó en total 20 ítemes-presa consumidos, todos caracterizados por ser mayoritariamente grandes invertebrados, entre los que se destacaron principalmente crustáceos (55\%) y moluscos (40\%) (Fig. 3a). Los ítemes más representativos en el contenido estomacal correspondieron a Homalaspis plana el que alcanzó un \%IRI de 71,4, seguido por Taliepus dentatus alcanzando un \%IRI de 12,5 y finalmente dos especies de Fissurella (crassa y nigra) ambas presentando un \%IRI de 2. En tanto, Allopetrolisthes angulosus, Chiton magnificus, Concholepas concholepas y Petrolisthes tuberculosus presentaron \%IRI bajos, cercanos a 0,1 (Tabla 2).

TABLA 1. Total de especies, longitud total $(\mathrm{cm})$, peso total $(\mathrm{g})$ y porcentaje de estómagos analizados.

TABLE 1. Total species, total length $(\mathrm{cm})$, total weight $(\mathrm{g})$ and percentage of stomachs analyzed.

\begin{tabular}{|c|c|c|c|c|c|c|}
\hline \multirow{2}{*}{ EsPeCIES } & \multirow{2}{*}{$\mathrm{N}$} & \multicolumn{2}{|c|}{ LONG TOTAL $(\mathrm{cm})$} & \multicolumn{2}{|c|}{ Peso total (g) } & \multirow{2}{*}{$\begin{array}{c}\% \\
\text { estómago } \\
\text { con } \\
\text { contenido }\end{array}$} \\
\hline & & Máx & Min & Max & Min & \\
\hline Pinguipes chilensis & 41 & 47 & 29 & 2500 & 400 & $95 \%$ \\
\hline Cheilodactylus variegatus & 38 & 33.5 & 27 & 1300 & 500 & $100 \%$ \\
\hline Aplodactylus punctatus & 32 & 38 & 25 & 1600 & 400 & $100 \%$ \\
\hline
\end{tabular}

(A) P. chilensis

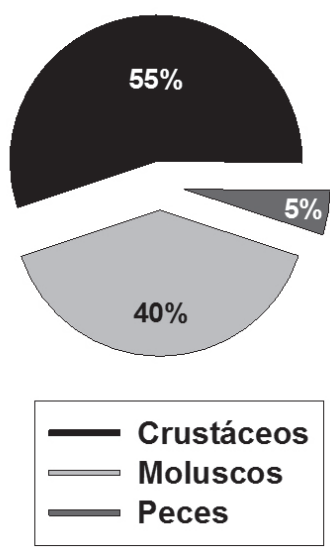

(B) Ch. variegatus
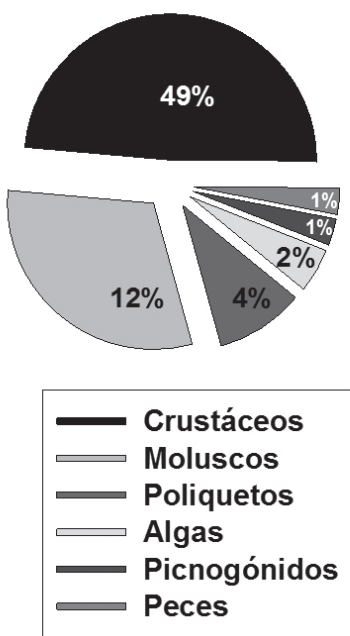

(C) A. punctatus

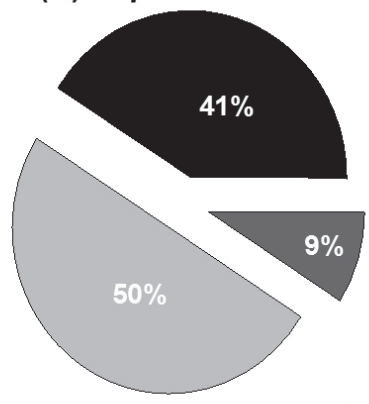

Algas Pardas

Algas Rojas

Algas Verdes

Figura 3. Porcentaje de preferencia de los diferentes grupos consumidos por A) P. chilensis, B) Ch. variegatus y C) A. punctatus, en noviembre del 2010.

Figure 3. Percentage of preference of different groups consumed by A) P. chilensis, B) Ch variegatus and C) A. punctatus, in November 2010. 
Para la especie Ch. variegatus, se analizaron en total 35 estómagos en los que se identificaron 37 ítemes-presa consumidos, todos caracterizados por ser principalmente pequeños invertebrados entre los que se destacan crustáceos (49\%), moluscos (31\%) y anélidos (10\%) (Fig. 3b). Los principales ítemes consumidos fueron anfípodos de la Familia Phoxocephalidae, seguido de poliquetos de la Familia Nereidae y en tercer lugar anfípodos pertenecientes a la Familia Eusiridae. Los poliquetos de la Familia Nereidae alcanzaron valores \%IRI del 23,2 del total de los ítemespresa analizados, mientras que las Familias de anfípodos Phoxocephalidae y Eusiridae alcanzaron valores \%IRI de 23,1 y 9,2 , respectivamente. Ítemes de menor representatividad, presentando valores porcentuales menores a 0,001 fueron Chiton magnificus, Choromytilus chorus, Fissurella picta, Liopetrolisthes mitra, Petrolisthes laevigatus, Petrolisthes tuberculosus y Venus antiqua (Tabla 3).
Para A. punctatus se analizaron 32 estómagos. Se identificó un total de 34 géneros como ítemes-presas consumidos, todos caracterizados por ser mayoritariamente algas rojas $(50 \%)$, pardas $(41 \%)$ y verdes $(9 \%)$ (Fig. 3c). El principal ítem consumido fue el alga parda Colpomenia sp., la que alcanzó un \%IRI de 32,4, seguida de las algas rojas Hymenena sp. y Bostrichya sp., las que alcanzaron un \%IRI de 23 y 11,5 respectivamente. Los valores más bajos de \%IRI fueron registrados por los géneros Desmarestia sp. y Ballia sp. los cuales no lograron superar el 0,001 del total de ítemes analizados (Tabla 4).

SOBREPOSICIÓN DIETARIA

El análisis de sobreposición de dieta entre las tres especies mostró una baja similitud entre las dietas. En el caso de $P$. chilensis y Ch. variegatus se presento una sobreposición alimentaria de 0,24 . Mientras que entre $P$. chilensis y $A$. punctatus y Ch. variegatus y A. punctatus la sobreposición fue mucho menor (Tabla 5).

TABLA 2. Porcentaje de ocurrencia (\%NO), frecuencia de ocurrencia (\%FO), índice de importancia relativa (IRI) y porcentaje IRI (\%IRI) de las presas consumidas por $P$. chilensis.

TABLE 2. Percentage of occurrence number ( $\%$ NO), frequency of occurrence ( $\%$ FO), index of relative importance (IRI) and IRI percentage (\% IRI) of prey consumed by P. chilensis.

\begin{tabular}{|c|c|c|c|c|c|}
\hline & \multirow{2}{*}{ ITEM-PRESA } & \multicolumn{3}{|c|}{ Pinguipes chilensis } & \multirow[b]{2}{*}{$\%$ IRI } \\
\hline & & $\% \mathrm{NO}$ & $\% \mathrm{FO}$ & IRI & \\
\hline \multirow[t]{10}{*}{ Crustacea } & Allopetrolisthes angulosus & 0,70423 & 1,13636 & 0,00800 & 0 \\
\hline & Allopetrolisthes spinifrons & 3,52113 & 3,40909 & 0,12004 & 1 \\
\hline & Cangrejo no identificado & 0,70423 & 1,13636 & 000800 & 0 \\
\hline & Homalaspis plana & 31,69014 & 31,81818 & 10,08323 & 72 \\
\hline & Leptograpsus variegatus & 5,63380 & 7,95455 & 0,44814 & 3 \\
\hline & Petrolisthes granulosus & 2,81690 & 4,54545 & 0,12804 & 1 \\
\hline & Petrolisthes laevigatus & 3,52113 & 3,40909 & 0,12004 & 1 \\
\hline & Petrolisthes tuberculatus & 2,81690 & 2,27273 & 0,06402 & 0 \\
\hline & Petrolisthes tuberculosus & 1,40845 & 1,13636 & 0,01601 & 0 \\
\hline & Taliepus dentatus & 15,49296 & 11,6364 & 1,76056 & 13 \\
\hline \multirow[t]{8}{*}{ Molusca } & Chaeteopleura peruviana & 3,52113 & 4,54545 & 0,16005 & 1 \\
\hline & Chiton magnificus & 0,70423 & 1,13636 & 0,00800 & 0 \\
\hline & Concholepas concholepas & 0,70423 & 1,13636 & 0,00800 & 0 \\
\hline & Fissurella crassa & 5,63380 & 4,54545 & 0,25608 & 2 \\
\hline & Fissurella nigra & 6,33803 & 4,54545 & 0,28809 & 2 \\
\hline & Fissurella picta & 2,11268 & 1,13636 & 0,02401 & 0 \\
\hline & Gaimardia trapesina & 2,81690 & 2,27273 & 0,06402 & 0 \\
\hline & Nacella clypeater & 5,63380 & 6,81818 & 0,38412 & 3 \\
\hline Pisces & Peces no identificados & 1,40845 & 2,27273 & 0,03201 & 1 \\
\hline
\end{tabular}


TABLA 3. Porcentaje de ocurrencia numérica (\%NO), frecuencia de ocurrencia (\%FO), índice de importancia relativa (IRI) y porcentaje IRI (\%IRI) de las presas consumidas por Ch. variegatus.

TABLE 3. Percentage of occurrence ( $\%$ NO), frequency of occurrence ( $\%$ FO), index of relative importance (IRI) and IRI percentage ( $\%$ IRI) of prey consumed by Ch. variegatus.

\begin{tabular}{|c|c|c|c|c|c|}
\hline & \multirow{2}{*}{ ITEM-PRESA } & \multicolumn{3}{|c|}{ Cheilodactylus variegatus } & \multirow[b]{2}{*}{$\%$ IRI } \\
\hline & & $\% \mathrm{NO}$ & $\% \mathrm{FO}$ & IRI & \\
\hline Arthropoda & Pycnogonida & 0,03692 & 0,72289 & 0,00027 & 0 \\
\hline \multirow[t]{20}{*}{ Crustacea } & Acanthocyclus gayi & 0,02769 & 0,24096 & 0,00007 & 0 \\
\hline & Anfipodos no identificados & 20,99142 & 6,98795 & 1,46687 & 22 \\
\hline & Anthuridae & 0,16616 & 1,44578 & 0,00240 & 0 \\
\hline & Aoridae & 0,47078 & 1,68675 & 0,00794 & 0 \\
\hline & Cancer setosus & 0,09231 & 1,68675 & 0,00156 & 0 \\
\hline & Cangrejo no identificado & 0,34155 & 2,65060 & 0,00905 & 0 \\
\hline & Eusiridae & 8,98181 & 6,74699 & 0,60600 & 9 \\
\hline & Gammaridae & 0,31386 & 0,48193 & 0,00151 & 0 \\
\hline & Homalaspis plana & 0,44309 & 4,57831 & 0,02029 & 0 \\
\hline & Ischyroceridae & 5,81556 & 7,22892 & 0,42040 & 6 \\
\hline & Isopodo no identificados & 0,08308 & 2,16867 & 0,00180 & 0 \\
\hline & Liopetrolisthes mitra & 0,00923 & 0,24096 & 0,00002 & 0 \\
\hline & Lyssianacidae & 3,26779 & 6,26506 & 0,20473 & 4 \\
\hline & Munna chilensis & 6,02788 & 8,19277 & 0,49385 & 8 \\
\hline & Petrolisthes laevigatus & 0,00923 & 0,24096 & 0,00002 & 0 \\
\hline & Petrolisthes tuberculatus & 0,07385 & 1,20482 & 0,00089 & 0 \\
\hline & Petrolisthes tuberculosus & 0,00923 & 0,24096 & 0,00002 & 0 \\
\hline & Phoxocephalidae & 24,28690 & 6,26506 & 1,52159 & 23 \\
\hline & Taliepus dentatus & 0,44309 & 3,37349 & 0,01495 & 0 \\
\hline & Tanaidaceo & 0,38770 & 2,16867 & 0,00841 & 0 \\
\hline \multirow[t]{12}{*}{ Molusca } & Aulacomya ater & 0,12000 & 1,20482 & 0,00145 & 0 \\
\hline & Chateopleura peruviana & 0,51694 & 3,85542 & 0,01993 & 0 \\
\hline & Chiton magnificus & 0,00923 & 0,24096 & 0,00002 & 0 \\
\hline & Chiton no identificado & 0,05539 & 0,96386 & 0,00053 & 0 \\
\hline & Choromytilus chorus & 0,00923 & 0,24096 & 0,00002 & 0 \\
\hline & Collisela zabrina & 0,05539 & 0,72289 & 0,00040 & 0 \\
\hline & Fissurella crassa & 0,48925 & 3,61446 & 0,01768 & 0 \\
\hline & Fissurella nigra & 0,07385 & 0,24096 & 0,00018 & 0 \\
\hline & Fissurella picta & 0,00923 & 0,24096 & 0,00002 & 0 \\
\hline & Gaimardia trapesina & 1,93852 & 2,40964 & 0,04671 & 1 \\
\hline & Tegula atra & 1,62467 & 5,06024 & 0,08221 & 1 \\
\hline & Venus antiqua & 0,00923 & 0,24096 & 0,00002 & 0 \\
\hline \multirow[t]{2}{*}{ Polychaeta } & Nereidae & 20,97295 & 12,04819 & 1,57733 & 23 \\
\hline & Prionospio sp. & 1,71698 & 3,13253 & 0,05378 & 1 \\
\hline Pisces & Peces no identificados & 0,10154 & 0,96386 & 0,00098 & 0 \\
\hline
\end{tabular}


TABLA 4. Porcentaje de ocurrencia numérica $(\% \mathrm{NO})$, frecuencia de ocurrencia (\%FO), índice de importancia relativa (IRI) y porcentaje IRI (\%IRI) de las presas consumidas por A. punctatus.

TABLE 4. Percentage of occurrence number (\% NO), frequency of occurrence (\% FO), index of relative importance (IRI) and IRI percentage (\% IRI) of prey consumed by $A$. punctatus.

\begin{tabular}{|c|c|c|c|c|c|c|}
\hline & & \multirow{2}{*}{ ITEM-PRESA } & \multicolumn{3}{|c|}{ Aplodactylus punctatus } & \multirow[b]{2}{*}{$\%$ IRI } \\
\hline & & & $\% \mathrm{VO}$ & $\% \mathrm{FO}$ & IRI & \\
\hline \multirow[t]{24}{*}{ Algae } & Phaeophyta & Adenocystis sp. & 0,00929 & 0,26178 & 0,00002 & 0 \\
\hline & & Colpomenia sp. & 29,35129 & 7,06806 & 2,07457 & 32 \\
\hline & & Desmarestia sp. & 0,02542 & 0,52356 & 0,00013 & 0 \\
\hline & & Durvillaea sp. & 0,00228 & 0,26178 & 0,00001 & 0 \\
\hline & & Lessonia sp. & 2,43742 & 4,45026 & 0,10847 & 2 \\
\hline & & Macrosystis sp. & 1,78868 & 2,09424 & 0,03746 & 1 \\
\hline & Rhodophyta & Ballia sp. & 0,00310 & 0,26178 & 0,00001 & 0 \\
\hline & & Bostrichya sp. & 9,69948 & 7,59162 & 0,73635 & 11 \\
\hline & & Callophylis sp. & 0,00929 & 0,52356 & 0,00005 & 0 \\
\hline & & Centroceras sp. & 0,48989 & 5,23560 & 0,02565 & 0 \\
\hline & & Ceramium sp. & 0,15656 & 2,87958 & 0,00451 & 0 \\
\hline & & Corallina sp. & 0,68279 & 2,35602 & 0,01609 & 0 \\
\hline & & Gelidium sp. & 2,94833 & 6,54450 & 0,19295 & 3 \\
\hline & & Grateoupia sp. & 2,00633 & 4,97382 & 0,09979 & 2 \\
\hline & & Hymenena sp. & 17,56858 & 8,37696 & 1,47171 & 23 \\
\hline & & Laurencia sp. & 2,67804 & 5,49738 & 0,14722 & 2 \\
\hline & & Mazzaela sp. & 1,96104 & 2,35602 & 0,04620 & 1 \\
\hline & & Polysiphonia sp. & 0,21961 & 1,04712 & 0,00230 & 0 \\
\hline & & Porphyra sp. & 2,52506 & 1,57068 & 0,03966 & 1 \\
\hline & & Sarcothalia sp. & 0,02688 & 0,26178 & 0,00007 & 0 \\
\hline & Chlorophyta & Codium sp. & 0,30482 & 3,14136 & 0,00958 & 0 \\
\hline & & Enteromorpha sp. & 1,88040 & 4,45026 & 0,08368 & 1 \\
\hline & & Ulva sp. & 4,58027 & 3,40314 & 0,15587 & 2 \\
\hline & & Algas no identificadas & 15,36837 & 6,80628 & 1,04601 & 16 \\
\hline \multirow[t]{2}{*}{ Crustacea } & & Cangrejo no identificado & 0,03421 & 0,26178 & 0,00009 & 0 \\
\hline & & Munna chilensis & 0,05653 & 2,61780 & 0,00148 & 0 \\
\hline \multirow[t]{5}{*}{ Molusca } & & Aulacomya ater & 1,10230 & 2,09424 & 0,02308 & 0 \\
\hline & & Fisurella sp.. & 0,00733 & 0,26178 & 0,00002 & 0 \\
\hline & & Gaimardia trapesina & 0,96545 & 6,54450 & 0,06318 & 1 \\
\hline & & Scurra scurra & 0,14451 & 0,26178 & 0,00038 & 0 \\
\hline & & Tegula atra & 0,11583 & 2,35602 & 0,00273 & 0 \\
\hline Polychaeta & & Phragmatopoma sp.. & 0,00538 & 0,52356 & 0,00003 & 0 \\
\hline Porifera & & Esponja no identificada & 0,17318 & 0,26178 & 0,00045 & 0 \\
\hline
\end{tabular}


TABLA 5. Valores de sobreposición alimentaria entre los ítems presa registrados en los contenidos estomacales de las tres especies de peces estudiadas.

TABLE 5. Values of diet overlap between prey items in the stomach contents of the three species studied.

\begin{tabular}{|c|c|c|}
\hline Especie & A. punctatus & Ch. variegatus \\
\hline P. chilensis & 0,01 & 0,24 \\
\hline A. punctatus & - & 0,09 \\
\hline
\end{tabular}

\section{DISCUSIÓN}

Las tres especies analizadas registraron diferencias en su contenido estomacal, presentando además una baja superposición trófica. Según Silva \& Stuardo (1985) las diferencias sobreposición en las dietas variaría según la oferta del hábitat. Estas especies se caracterizan por compartir el mismo ambiente, pero de diferente forma; $C h$. variegatus y A. punctatus habitarían entre los bosques de Macrosystis pirifera ("huiro"), los cuales ocupan como refugio, así como también muchas otras especies de pequeños peces e invertebrados, mientras que $P$. chilensis lo hace cerca de sustratos duros o blandos del fondo (Moreno \& Flores 2002), factor que provocaría las diferencias entre sus dietas.

Considerando las longitudes y pesos de los individuos muestreados, se observa que las tres especies se encontraron dentro del rango promedio de la talla máxima estipulada, de acuerdo a las guías de identificación de peces (Bahamonde \& Pequeño 1975), por lo que las tres especies analizadas corresponden mayoritariamente a ejemplares adultos.

P. chilensis presentó un amplio espectro trófico, compuesto principalmente por especies de gran tamaño, tanto sésiles como móviles, especialmente crustáceos, por lo que se confirma su naturaleza de depredador generalista (Moreno \& Zamorano 1980; González \& Oyarzun 2003). Dieta que según Medina et al. (2004) dependerá de la oferta alimentaria bentónica del hábitat frecuentado. La alta incidencia de crustáceos decápodos que se observó señalan a esta especie en primera instancia como carcinófaga (González \& Oyarzun 2003), y en forma secundaria consumidora de moluscos, coincidiendo con lo descrito con Vargas et al. (1999) para esta misma especie en la zona norte y por Silva \& Stuardo (1985) en la zona centro-sur, con variaciones en la preferencia de las especies presas.

P. chilensis presenta claras adaptaciones morfológicas para el tipo de alimento que requiere (i.e. dentadura bastante desarrollada, estómago bien definido con secreciones ácidos fuertes, e intestino corto), los cual sumado al gran tamaño de su boca, le permiten consumir un gran espectro de presas. Además, existe relación entre la forma corporal y la alimentación, ya que la mayoría de las presas encontradas en los estómagos son de gran longitud y poco evasivas, por lo que no requieren una velocidad de captura. La preferencia por presas de mayor tamaño, no es sólo porque son más fáciles de ver, sino que también son más provechosas en términos de energía neta ingerida (Bannon \& Ringler 1986). Así, el consumo de presas más beneficiosas, les permite compensar el alto gasto de energía que realizan en los procesos de producción de gametos, reproducción y crecimiento (Sano 1993).

Por otra parte, Ch. variegatus también es un depredador generalista, pero a diferencia de $P$. chilensis, es un depredador de tipo micrófago (i.e. animales que se alimentan de presas minúsculas), con una dieta muy diversificada, donde los pequeños crustáceos, poliquetos y moluscos son los más frecuentemente consumidos. Nuestros resultados confirman lo registrado por Vegas \& Rojas en el año 1987 , donde se reafirma el carácter carnívoro de esta especie, siendo las presas dominantes mitílidos y poliquetos. Sin embargo, en otro estudios en el litoral norte de Chile y Perú se postula a Ch. variegatus como una especie omnívora (Hoyos et al. 1985), por lo cual nosotros postulamos al igual que $P$. chilensis que la oferta alimenticia incidiría en la dieta de esta especie.

Ch. variegatus presenta adaptaciones para la obtención de su alimento, poseyendo una pequeña boca de posición subterminal que esta provista de notorios labios, la que influye en el tipo y tamaño de presa que consume, además al ser una especie carnívora en esta zona, también presenta un estomago bien definido con secreciones ácidas muy fuertes e intestino muy corto, que actúa sobre el alimento ya consumido.

Contrariamente a $P$. chilensis y Ch. variegatus, Aplodactylus punctatus es una especie mayoritariamente herbívora, las macroalgas constituyeron más del $95 \%$ de la biomasa total en la dieta y están presentes en el $100 \%$ de los estómagos analizados. Los patrones de selectividad de esta especie siguieron las tendencias descritas por otros autores para otras especies herbívoras (Montgomery \& Gerking 1980; 
Horn et al. 1982; Cáceres et al. 1993), caracterizados por el consumo preferencial de algas rojas. Este consumo por parte de peces herbívoros, ha sido atribuido a que este tipo de alga posee como compuesto de reserva el almidón florídeo (Bold \& Wynne 1978) un polímero de glucosa con enlaces alfa, que probablemente pueda ser hidrolizado por las enzimas digestivas de los teleósteos (Cáceres et al. 1993). Sin embargo, algas pardas y verdes también fueron registradas en los estómagos, pero con una menor representatividad, excepto el alto consumo de Colpomenia sp., particularmente abundante en la zona de estudio (Cáceres et al. 1993). Según varios autores, la baja preferencia de algas pardas, estaría mayoritariamente asociada a la baja digestibilidad que poseen, comparadas con las algas rojas y verdes (Montgomery \& Gerking 1980; Horn 1989), mientras que las algas verdes a pesar de su alto contenido de nitrógeno, energía y digestibilidad (Cáceres et al. 1993) su pobre presencia en los estómagos se deba probablemente a su baja disponibilidad ambiental en la zona estudiada. Además de la presencia de algas, también se registraron isópodos, briozoos y poliquetos en estómagos de A. punctatus, los cuales se encontraban pobremente digeridos, por lo que nosotros presumimos que su presencia solo responde a que son fauna acompañante del principal ítem presa de esta especie, ya que representan menos del 1\% de IRI.

Aplodactylus punctatus presenta una boca ligeramente ventral y provista de labios gruesos, con dientes tipo incisivos aplanados, formando tres cúspides en su extremo libre dispuestos en varias filas. Presenta un estómago bien definido con un $\mathrm{pH}$ marcadamente ácido y un intestino muy alcalino (Cáceres et al. 1993). La importancia del pH como mecanismo de lisis química de las paredes celulares de las algas ha sido sugerida por varios autores (Cáceres et al. 1993). El pH acido facilitaría la liberación de los nutrientes contenido dentro de las células, aun cuando el mecanismo especifico de acción permanece desconocido (Cáceres et al. 1993). La presencia de un intestino largo, característica de la mayoría de los peces herbívoros (Horn 1989), por una parte aumenta el tiempo de retención del alimento a la vez que incrementa la eficiencia en la extracción de los nutrientes por parte del animal (Silby 1981).

En conclusión, nosotros complementamos el conocimiento de la biología trófica en el sur de Chile de Pinguipes chilensis y Cheilodactylus variegatus confirmándolos como depredadores carnívoros generalistas, siendo esta última especie además considerada como micrófago, y Aplodactylus punctatus como una especie herbívora especialista con preferencia sobre algas rojas, encontrando diferencias entre sus dietas y con una pobre sobreposición dietaria.

\section{AGRADECIMIENTOS}

Este estudio ha sido financiado por el Proyecto FONDECYT 1110798: "Determinación de indicadores geográfico ambientales y de riesgo natural: Herramientas de soporte decisional para la planificación y gestión territorial en sistemas costeros".

\section{BIBLIOGRAFÍA}

Bahamonde, N. \& Pequeño, G. 1975. Peces de Chile. Lista sistemática. Publicación Ocasional, Museo Nacional de Historia Natural, Chile. 21:1-20.

Bannon, E. \& Ringler, N.H. 1986. Optimal prey size for stream resident brown trout (Salmo trutta): tests of predictive models. Canadian Journal of Zoology. 64:704-713.

BARNARD, J.L. 1969. The families and genera of marine Gammaridean Amphipoda. United States National Museum Bulletin. 271:50-481.

Bertrán, C., Jiménez, C., Fierro P., Peña-Cortés, F., Tapia, J., Hauenstein, E. \& Vargas-Chacoff, L. 2013. Alimentación de Micropogonias furnieri (Osteichthyes: Sciaenidae) en el lago costero Budi, Sur de Chile. Revista de Biología Marina y Oceanografía. 48:193-197.

Bold, H.C. \& Wynne, M.J. 1978. Introduction to the algae. Structure and reproduction. Prentice-Hall. Englewood Cliffs. $286 \mathrm{pp}$

Botero, M. 2004. Comportamiento de los peces en la búsqueda y la captura del alimento / Fishes feeding behavior. Revista colombiana de ciencias pecuarias.17(1):63-75

Cáceres, C., Benavides A. \& Ojeda, P. 1993. Ecología trófica del pez herbívoro Aplodactylus punctatus (Pisces: Aplodactylidae) en la costa centro-norte de Chile. Revista Chilena de Historia Natural. 66:185-194.

Chirichigno, N. 1998. Clave para identificar los peces marinos del Perú. Informe Instituto del Mar Perú, Callao. 44:1-387.

Fauchald, K. 1977. The polychaete worms. Definitions and keys to the Orders, families and genera. Natural History Museum of the Angeles County. Science Series. 28:1-190.

Fierro, P., Bertrán, C., Martínez, D., Valdovinos, C. \& VargasChacoff, L. 2014. Ontogenetic and temporal changes in the diet of the Chilean silverside Odontesthes regia (Atherinidae) in southern Chile. Cahiers de Biologie Marine. 55:323-332.

García, C. \& Chong, J. 2002. Composición de la dieta de Brama australis Valenciennes 1837 en la zona Centro-Sur de Chile (VIII Región) en Otoño 2000 y Verano 2001. Gayana Zoología. 66(2):225-230.

GonzÁlez, P. 2001. Biología reproductiva y conducta trófica de Pinguipes chilensis Valenciennes 1833 (Perciformes, Pinguipedidae). Tesis de Magíster. Universidad de Concepción, Concepción, Chile. 160 pp.

GonzÁlez, P. \& OYARZún, C. 2003. Diet of the Chilean sandperch, Pinguipes chilensis (Perciformes, Pinguipedidae) in 
southern Chile. Journal of Applied Ichthiology. 19:371375.

GrIFFITHS, M.H. 1997. Influence of prey availability on the distribution of dusky kob Argyrosomus japonicas (Sciaenidae) in the Great Fish River estuary, with notes on the diet of early juveniles from three other estuarine systems. South African Journal Marine Science. 18:137145.

HoRn, M.H. 1989. Biology of marine herbivorous fishes. Oceanography and Marine Biology: An Annual Review. 27:167-272.

Horn, M.H, Murray, S.N. \& Edwards, T.W. 1982. Dietary selectivity in the field and foog preferences in the laboratory for two herbivorous fishes (Cebidichikys violaceus and Xiphister mucosus) from a temperate intertidal zone. Marine Biology. 67:237-246.

Hoyos, L., Tarazona, J., Shiga, B. \& Chiang, V. 1985. Algunos cambios en la ictiofauna y sus relaciones tróficas durante el Fenómeno "El Niño" en la bahía de Ancón. En:. Arntz W, A Landa \& J Tarazona (eds). "El Niño" su impacto en la fauna marina. Boletín Instituto del Mar Perú-Callao. Boletín extraordinario:163-171.

Hyslop, E.J. 1980. Stomach contents analysis - a review of methods and their application. Journal of Fish Biology. 17:411-429.

JiMÉNEZ, C. 2006. Estructura dietaria del Hualquil Micropogonias furnieri (Desmarest 1823) en la laguna costera Budi, IX Región de la Araucanía. Tesis de Biología Marina. Universidad Austral de Chile. Valdivia, Chile. 88 pp.

Medina, M., Araya, M. \& Vega, C. 2004. Alimentación y relaciones tróficas de peces costeros de la zona norte de Chile. Investigaciones Marinas. 32(1):33-47.

Menzies, R. 1962. The zoogeography, ecology, and systematics of the Chilean marine isopods. Lamont geological observatory Columbia University, New York. 162 pp.

Montgomery, W.L., Myrberg, A.A. \& Fishelson, L. 1989. Feeding ecology of surgeonfishes (Acanthuridae) in the Northerm Red Sea, with particular reference to Acanthurus nigrofscus (Forsskal). Journal of Experimental Marine Biology and Ecology. 32:179-207.

Moreno, C. \& Zamorano, J. 1980. Selectividad del alimento en dos peces bentófagos (Mugiloides chilensis y Calliclinus geniguttatus). Boletim do Instituto Oceanográfico, Sao Paulo. 29(2):245-249.

Moreno, M. \& Flores, H. 2002. Contenido estomacal de Cheilodactylus variegatus Valenciennes 1833, Pinguipes chilensis Valenciennes 1833 y Prolatilus jugularis Valenciennes 1833 en la Bahia de la Herradura, Coquimbo, durante primavera del 2001. Gayana 66(2):213-217.

Navarro, J. \& Pequeño, G. 1979. Peces litorales de los Archipiélagos de Chiloé y Los Chonos, Chile. Revista de Biología Marina. 16(3):255-309.

Pequeño, G. 2001. El "Bilagay" (Cheilodactylus variegatus Valenciennes, 1833), en las costas de Valdivia (Osteichthyes: Cheilodactylidae). Estudios Oceanológicos. 20:23-28.
Pequeño, G. \& Lamilla, J. 1995. Peces intermareales de la costa de Llanquihue (Chile): composición taxonómica, abundancia relativa y gradiente de distribución longitudinal. Revista de Biología Marina. 30(1):7-27

Pequeño, G., Lamilla. J., Lloris, D. \& Rucabado, J. 1995. Comparación entre las ictiofaunas intermareales de los extremos austral y boreal de los canales patagónicos. Revista de Biología Marina. 30 (2):155-177.

PiankA, E. 1973. The structure of lizard communities. Annual Review of Ecology \& Systematics. 4:53-74.

Pinkas, L., Oliphant, M.S. \& Iverson, Y.L.K. 1971. Foods habits of albacore, bluefin tuna and bonito in California waters. California Department of Fish and Game. Fish Bulletin. 152:1-105.

SANO, M. 1993. Foraging activities and diets of males and females in a haremic sandperch (Pisces: Pinguipedidae). Marine Ecology Progress Series. 98:55-59.

SAntelices, B. 1989. Algas marinas de Chile. Edición Universidad Católica de Chile. Santiago. 339 pp.

SERNAPESCA. 2011. Anuario Estadístico de Pesca y Acuicultura. Servicio Nacional de Pesca. Ministerio de Economía Fomento y Reconstrucción, Valparaíso. 240 pp.

Siegel, S. \& Castellan, N.J. 1988. Nonparametrics statistica for the behavioral sciences, McGraw-Hill Book Company, New York. 399 pp.

Silby, R.M. 1981. Strategies of digestion and defecation. In: Physiological ecology: An evolutionary approach to resource use (Eds. Townsend, C.R. \& P. Calow), pp. 109139. Blackwell Scientific Publications, London.

Silva, M. \& Stuardo, J. 1985. Alimentación y relaciones tróficas generales entre algunos peces demersales y el bentos de Bahía Coliumo (Provincia de Concepción, Chile). Gayana Zoología. 49:77-102.

Unesco. 1968. Zooplankton sampling, Imprimerie Rolland, Paris. $174 \mathrm{pp}$.

Vargas, L. \& Pequeño, G. 2001. Hallazgo del Bilagay (Cheilodactylus variegatus Valenciennes, 1833), en la Bahia Metri, Chile. (Osteichthyes: Cheilodactylidae). Investigaciones Marinas. 29(2):25-37.

Vargas, M.E, Soto, R.A. \& Guzmán, G.L. 1999. Cambios interanuales en la alimentación de peces submareales del Norte de Chile entre los $20^{\circ} 11^{\prime}$ y $20^{\circ} 20^{\prime} \mathrm{S}$. Revista de Biología Marina y Oceanografía. 34(2):197-210.

Vegas, M. \& Rojas, E. 1987. Estudio de algunos peces marinos relacionados con el litoral rocoso del Perú. $2^{\circ}$ Congreso Latinoamericano sobre Ciencias del Mar. Anales Científicos, Universidad Nacional Agraria La Molina, Lima. 11:249-263.

ZaGal, C. \& Hermosilla, C. 2001. Guía de invertebrados marinos del litoral Valdiviano. Quebecor World Chile, Santiago de Chile. 217 pp.

Zaret, T. \& Paine, R.T. 1973. Species introduction in a tropical lake. Science. 182:449-455.

Recibido: 06.01.14

Aceptado: 21.11.14 